\title{
REMARKS ON SOME INTEGRALS AND SERIES INVOLVING THE STIRLING NUMBERS AND $\zeta(n)$
}

\author{
LI-CHIEN SHEN
}

ABSTRACT. From the perspective of the well-known identity

$$
{ }_{2} F_{1}(a, b ; c ; 1)=\frac{\Gamma(c) \Gamma(c-a-b)}{\Gamma(c-a) \Gamma(c-b)},
$$

we clarify the connections between the Stirling numbers $s_{k}^{n}$ and the Riemann zeta function $\zeta(n)$. As a consequence, certain series and integrals can be evaluated in terms of $\zeta(n)$ and $s_{k}^{n}$.

\section{INTRODUCTION}

In a recent paper [2], applying Parseval's identity to a Fourier series and the contour integral to a generating function, D. Borwein and J. M. Borwein established the following interesting identities:

$$
\begin{gathered}
\sum_{n=1}^{\infty} \frac{1}{(n+1)^{2}}\left(\sum_{k=1}^{n} \frac{1}{k}\right)^{2}=\frac{11}{4} \zeta(4), \\
\sum_{n=1}^{\infty} \frac{1}{n^{2}}\left(\sum_{k=1}^{n} \frac{1}{k}\right)^{2}=\frac{17}{4} \zeta(4),
\end{gathered}
$$

and

$$
\sum_{n=1}^{\infty} \frac{1}{n^{3}}\left(\sum_{k=1}^{n} \frac{1}{k}\right)=\frac{5}{4} \zeta(4) .
$$

In this paper, we will approach the infinite series of this type from a different angle. In addition to providing a different proof to (i), we also derive the following identities of a similar nature:

$$
\sum_{n=1}^{\infty} \frac{1}{(n+1)^{2}}\left(\sum_{k=1}^{n} \frac{1}{k}\right)=\zeta(3)
$$

Received by the editors August 30, 1993 and, in revised form, January 17, 1994; originally communicated to the Proceedings of the AMS by Albert Baernstein II.

1991 Mathematics Subject Classification. Primary 11M99, 33B15.

Key words and phrases. Riemann zeta function, hypergeometric series, Stirling numbers of the first kind. 


$$
\begin{aligned}
\sum_{n=1}^{\infty} \frac{1}{(n+1)^{2}}\left(\sum_{k=1}^{n} \frac{1}{k^{2}}\right) & =\frac{3}{4} \zeta(4), \\
\sum_{n=1}^{\infty} \frac{1}{(n+1)^{3}}\left(\sum_{k=1}^{n} \frac{1}{k}\right) & =\frac{1}{4} \zeta(4) .
\end{aligned}
$$

Furthermore, in the process we will see the close connection between $\zeta(n)$ and the Stirling numbers of the first kind. It should be stressed that the identities (iv), (v) and (vi) are undoubtedly known. For example, Bruce Berndt gives a brief account on the identity (iv) in [1, p. 251] which, according to him, was first discovered by Euler in 1775 .

Essentially, the key ingredient of our paper is based on the following wellknown identity [5, p. 282]

$$
{ }_{2} F_{1}(a ; b ; c ; 1)=\frac{\Gamma(c) \Gamma(c-a-b)}{\Gamma(c-a) \Gamma(c-b)}, \quad \operatorname{Re}(c-a-b)>0 .
$$

We will view the quantities in both sides of the above identity as analytic functions of the variable $a$. Then, by equating the coefficients of their power series, we obtain a collection of identities, and among them are (iv) and (v). In Section 2 , we will treat the special case in which $a=b$ and $c=1$ due to its unusual simplicity. The general case is treated in Section 3.

\section{Proofs OF IDENTITIES}

We begin with the familiar binomial theorem

$$
(1-z)^{-\alpha}=\sum_{n=0}^{\infty} \frac{(\alpha)_{n}}{n !} z^{n}, \quad|z|<1,
$$

where $(\alpha)_{n}=\alpha(\alpha+1)(\alpha+2) \cdots(\alpha+n-1)$. Letting $\alpha$ be real and $z=r e^{i \theta}$, $0 \leq r<1$, we obtain from Bessel's equality that

$$
\frac{1}{2 \pi} \int_{0}^{2 \pi}\left|1-r e^{i \theta}\right|^{-2 \alpha} d \theta=\sum_{n=0}^{\infty}\left(\frac{(\alpha)_{n}}{n !}\right)^{2} r^{2 n}
$$

From now on, we restrict ourselves to the case $\alpha<\frac{1}{2}$ in which the integral in (2) remains finite when $r=1$. Now letting $r \rightarrow 1^{-}$, we obtain

$$
\frac{1}{2 \pi} \int_{0}^{2 \pi}\left|1-e^{i \theta}\right|^{-2 \alpha} d \theta=\sum_{n=0}^{\infty}\left(\frac{(\alpha)_{n}}{n !}\right)^{2} \quad \text { for } \alpha<\frac{1}{2} .
$$

Since $\left|1-e^{i \theta}\right|=2 \sin \frac{\theta}{2}$, we have

$$
\begin{aligned}
\int_{0}^{2 \pi}\left|1-e^{i \theta}\right|^{-2 \alpha} d \theta & =2^{-2 \alpha} \int_{0}^{2 \pi} \sin ^{-2 \alpha} \frac{\theta}{2} d \theta=2^{-2 \alpha+2} \int_{0}^{\frac{\pi}{2}} \sin ^{-2 \alpha} \theta d \theta \\
& =2^{-2 \alpha+1} \sqrt{\pi} \Gamma\left(\frac{1}{2}-\alpha\right) / \Gamma(1-\alpha) .
\end{aligned}
$$

Thus, we have, for $\alpha<\frac{1}{2}$,

$$
\sum_{n=0}^{\infty}\left(\frac{(\alpha)_{n}}{n !}\right)^{2}=\frac{1}{2 \pi} \int_{0}^{2 \pi}\left|1-e^{i \theta}\right|^{-2 \alpha} a \theta=\frac{2^{-2 \alpha}}{\sqrt{\pi}} \frac{\Gamma\left(\frac{1}{2}-\alpha\right)}{\Gamma(1-\alpha)}
$$


In the following discussions, we will view the three quantities of the above identity as functions of $\alpha$; in particular, we note that they are analytic at $\alpha=0$ and, thus, can be expressed in terms of the power series at $\alpha=0$.

We begin with the integral in (4) by writing

$$
\begin{aligned}
\left|1-e^{i \theta}\right|^{-2 \alpha} & =\exp \left(-2 \alpha \ln \left|1-e^{i \theta}\right|\right) \\
& =\sum_{n=0}^{\infty} \frac{(-1)^{n} 2^{n} \alpha^{n}}{n !}\left(\ln \left|1-e^{i \theta}\right|\right)^{n}, \quad \theta \neq 0 ;
\end{aligned}
$$

then

$$
\frac{1}{2 \pi} \int_{0}^{2 \pi}\left|1-e^{i \theta}\right|^{-2 \alpha} d \theta=\sum_{n=0}^{\infty} \frac{(-1)^{n} 2^{n}}{n !}\left(\frac{1}{2 \pi} \int_{0}^{2 \pi} \ln ^{n}\left|1-e^{i \theta}\right| d \theta\right) \alpha^{n} .
$$

Next, we deal with the third quantity in (4). Let

$$
f(z)=\frac{2^{-2 z}}{\sqrt{\pi}} \frac{\Gamma\left(\frac{1}{2}-z\right)}{\Gamma(1-z)}=\sum_{n=0}^{\infty} a_{n} z^{n} .
$$

Since $\Gamma\left(\frac{1}{2}\right)=\sqrt{\pi}$, it is easy to see that $a_{0}=1$. Our immediate goal is to deduce the following simple recursion formula for $a_{n}$ :

$$
a_{1}=0 \text { and }(n+1) a_{n+1}=2 \sum_{k=1}^{n} a_{n-k}\left(2^{k}-1\right) \zeta(k+1) \text { for } n \geq 1 .
$$

Thus

$$
\begin{aligned}
& a_{2}=\zeta(2), \quad a_{3}=2 \zeta(3), \\
& a_{4}=\frac{1}{2}\left(\zeta^{2}(2)+7 \zeta(4)\right)=\frac{19}{4} \zeta(4), \\
& a_{5}=2 \zeta(2) \zeta(3)+6 \zeta(5) .
\end{aligned}
$$

So the coefficients of $f(z)$ are essentially the sums of the products of $\zeta(n)$.

The derivation of $(6)$ hinges on the following identity:

$$
\frac{f^{\prime}}{f}(z)=2 \sum_{n=1}^{\infty}\left(2^{n}-1\right) \zeta(n+1) z^{n} .
$$

Before proving (8), we first see that (6) can be derived from it as follows:

$$
\begin{aligned}
\sum_{n=0}^{\infty}( & +1) a_{n+1} z^{n}=f^{\prime}(z)=\frac{f^{\prime}(z)}{f(z)} \cdot f(z) \\
& =2\left(\sum_{n=1}^{\infty}\left(2^{n}-1\right) \zeta(n+1) z^{n}\right) \cdot\left(\sum_{n=0}^{\infty} a_{n} z^{n}\right) \\
& =2 \sum_{n=1}^{\infty}\left(\sum_{k=1}^{n} a_{n-k}\left(2^{k}-1\right) \zeta(k+1)\right) z^{n} .
\end{aligned}
$$

And equating the corresponding coefficients readily yields (6).

We now prove (8). First we recall that the Riemann zeta function $\zeta(s)$ satisfies

$$
\sum_{n=0}^{\infty} \frac{1}{(2 n+1)^{s}}=\left(1-\frac{1}{2^{s}}\right) \zeta(s), \text { for } \operatorname{Re} s>1
$$


We note that

$$
\sum_{n=0}^{\infty}\left(\frac{1}{\left(n+\frac{1}{2}\right)}-\frac{1}{(n+1)}\right)=2 \sum_{n=0}^{\infty}\left(\frac{1}{2 n+1}-\frac{1}{2 n+2}\right)=2 \ln 2 .
$$

We also need the following power series expansion at $z=0$ :

$$
\begin{aligned}
& \frac{1}{n+\frac{1}{2}-z}-\frac{1}{n+1-z}=\sum_{k=0}^{\infty}\left(\frac{1}{\left(n+\frac{1}{2}\right)^{k+1}}-\frac{1}{(n+1)^{k+1}}\right) z^{k} \\
& \quad=\frac{1}{n+\frac{1}{2}}-\frac{1}{n+1}+\sum_{k=1}^{\infty}\left(\frac{2^{k+1}}{(2 n+1)^{k+1}}-\frac{1}{(n+1)^{k+1}}\right) z^{k}
\end{aligned}
$$

Hence,

$$
\begin{aligned}
& \sum_{n=0}^{\infty}\left(\frac{1}{n+\frac{1}{2}-z}-\frac{1}{n+1-z}\right) \\
& \quad=2 \ln 2+\sum_{k=1}^{\infty}\left(\frac{2^{k+1}}{(2 n+1)^{k+1}}-\frac{1}{(n+1)^{k+1}}\right) z^{k} \\
& \quad=2 \ln 2+2 \sum_{k=1}^{\infty}\left(2^{k}-1\right) \zeta(k+1) z^{k} \quad(\text { from (9)) }
\end{aligned}
$$

The most crucial part of the proof depends on the infinite product identity for $\frac{1}{\Gamma(z)}[5$, p. 236]:

$$
\frac{1}{\Gamma(z)}=z e^{-\gamma z} \prod_{n=1}^{\infty}\left(1+\frac{z}{n}\right) e^{-z / n}
$$

Differentiating $\frac{1}{\Gamma(z)}$ logarithmically and replacing $z$ by $a-z$, we have

$$
\frac{\Gamma^{\prime}}{\Gamma}(a-z)=-\gamma-\frac{1}{a-z}+\sum_{n=1}^{\infty}\left(\frac{1}{n}-\frac{1}{n+a-z}\right) .
$$

From (12), we see that

$$
\begin{aligned}
\frac{f^{\prime}}{f}(z) & =-2 \ln 2+\frac{\Gamma^{\prime}}{\Gamma}(1-z)-\frac{\Gamma^{\prime}}{\Gamma}\left(\frac{1}{2}-z\right) \\
& =-2 \ln 2+\sum_{n=0}^{\infty}\left(\frac{1}{n+\frac{1}{2}-z}-\frac{1}{n+1-z}\right) \\
& =2 \sum_{k=1}^{\infty}\left(2^{k}-1\right) \zeta(k+1) z^{k} \quad(\text { from }(11)) .
\end{aligned}
$$

This completes the proof of (8).

We now come to the infinite sum in (4). We note first that $(\alpha)_{n}=\prod_{k=0}^{n-1}(\alpha+k)$ is a polynomial of degree $n$ in $\alpha$, and it is often written as

$$
(\alpha)_{n}=\sum_{k=1}^{n} s_{k}^{n} \alpha^{k}
$$


where $s_{k}^{n}$ are known as the Stirling numbers of the first kind (for more details of their properties, see [3, p. 43]). It is easy to see that

$$
\frac{s_{1}^{n}}{n !}=\frac{1}{n} \quad \text { and } \quad \frac{s_{2}^{n}}{n !}=\frac{1}{n} \sum_{k=1}^{n-1} \frac{1}{k} .
$$

To obtain a recursion formula for $s_{k}^{n}$, we differentiate (14) logarithmically and repeat the same argument as we did earlier for the derivation of (8). Then

$$
(k-1) s_{k}^{n}=\sum_{m=1}^{k-1} s_{k-m}^{n} A_{m}^{n},
$$

where $A_{0}^{1}=1, A_{m}^{1}=0$ for $m \geq 1$ and

$$
A_{m}^{n}=(-1)^{m+1} \sum_{k=1}^{n-1} \frac{1}{k^{m}} \quad \text { for } n \geq 2 \text {. }
$$

Thus, from (15),

$$
\begin{aligned}
& \frac{s_{3}^{n}}{n !}=\frac{1}{2 n}\left\{\left(\sum_{k=1}^{n-1} \frac{1}{k}\right)^{2}-\sum_{k=1}^{n-1} \frac{1}{k^{2}}\right\}, \\
& \frac{s_{4}^{n}}{n !}=\frac{1}{6 n}\left\{\left(\sum_{k=1}^{n-1} \frac{1}{k}\right)^{3}-2\left(\sum_{k=1}^{n-1} \frac{1}{k}\right)\left(\sum_{k=1}^{n-1} \frac{1}{k^{2}}\right)+\sum_{k=1}^{n-1} \frac{1}{k^{3}}\right\} .
\end{aligned}
$$

From (14), we have

$$
\begin{aligned}
& \left(\frac{(\alpha)_{n}}{n !}\right)^{2}=\sum_{k=2}^{2 n} \sigma_{k}^{n} \alpha^{k} \quad \text { for } n \geq 1 \text { and } \\
& \sum_{n=0}^{\infty}\left(\frac{(\alpha)_{n}}{n !}\right)^{2}=1+\sum_{k=2}^{\infty}\left(\sum_{n \geq \frac{k}{2}}^{\infty} \sigma_{k}^{n}\right) \alpha^{k},
\end{aligned}
$$

where

$$
\sigma_{k}^{n}=\sum_{m=1}^{k-1} \frac{s_{k-m}^{n}}{n !} \frac{s_{m}^{n}}{n !} .
$$

The first few $\sigma_{n}$ are quite easy to compute and they are

$$
\begin{aligned}
& \sigma_{2}^{n}=\frac{1}{n^{2}}, \quad \sigma_{3}^{n}=\frac{2}{n^{2}} \sum_{k=1}^{n-1} \frac{1}{k} \text { and } \\
& \sigma_{4}^{n}=\frac{2 s_{3}^{n}}{n !} \frac{s_{1}^{n}}{n !}+\left(\frac{s_{2}^{n}}{n !}\right)^{2}=\frac{1}{n^{2}}\left(2\left(\sum_{k=1}^{n-1} \frac{1}{k}\right)-\sum_{k=1}^{n-1} \frac{1}{k^{2}}\right) .
\end{aligned}
$$

Now using (4) and comparing the coefficients of the series (5) and (17), we have 


\section{Proposition 1.}

$$
\frac{1}{2 \pi} \int_{0}^{2 \pi} \ln ^{k}\left|1-e^{i \theta}\right| d \theta=(-1)^{k} \frac{k !}{2^{k}} \sum_{n \geq \frac{k}{2}}^{\infty} \sigma_{k}^{n} .
$$

And for $k=1,2,3,4$, we can conclude more specifically from (5), (7) and (18) the following

\section{Corollary.}

$$
\begin{gathered}
\frac{1}{2 \pi} \int_{0}^{2 \pi} \ln \left|1-e^{i \theta}\right| d \theta=0 \\
\frac{1}{2 \pi} \int_{0}^{2 \pi} \ln ^{2}\left|1-e^{i \theta}\right| d \theta=\frac{1}{2} \zeta(2) \\
\frac{1}{2 \pi} \int_{0}^{2 \pi} \ln ^{3}\left|1-e^{i \theta}\right| d \theta=-\frac{3}{2} \sum_{n=2}^{\infty} \frac{1}{n^{2}}\left(\sum_{k=1}^{n-1} \frac{1}{k}\right)=-\frac{3}{2} \zeta(3)
\end{gathered}
$$

and

$$
\begin{aligned}
\frac{1}{2 \pi} \int_{0}^{2 \pi} \ln ^{4}\left|1-e^{i \theta}\right| d \theta & =3 \sum_{n=2}^{\infty} \frac{1}{n^{2}}\left(\sum_{k=1}^{n-1} \frac{1}{k}\right)^{2}-\frac{3}{2} \sum_{n=2}^{\infty} \frac{1}{n^{2}}\left(\sum_{k=1}^{n-1} \frac{1}{k^{2}}\right) \\
& =\frac{57}{8} \zeta(4) .
\end{aligned}
$$

Remarks. The identity $(20)$ is well known and plays an important role in the proof of Jensen's theorem [4, p. 299].

The identity (22) yields (iv):

$$
\zeta(3)=\sum_{n=1}^{\infty} \frac{1}{(n+1)^{2}}\left(\sum_{k=1}^{n} \frac{1}{k}\right),
$$

and this is an interesting comparison with

$$
2 \zeta(3)=\sum_{n=1}^{\infty} \frac{1}{n^{2}}\left(\sum_{k=1}^{n} \frac{1}{k}\right) .
$$

And, from (23) and (i), we deduce (v):

$$
\sum_{n=1}^{\infty} \frac{1}{(n+1)^{2}}\left(\sum_{k=1}^{n} \frac{1}{k^{2}}\right)=\frac{3}{4} \zeta(4) .
$$

In the next section, we will provide a different proof of (i).

\section{Further EXTENSIONS}

In this section, we will apply the method developed in the previous section to the identity (1) to obtain additional identities. Since the procedures are identical, we will omit all the details. As before, we regard quantities in (1) as functions of $a$ and the goal is to obtain their power series expansions at $a=0$. 
First, we replace $a$ in (1) by $z$. Then

$$
{ }_{2} F_{1}(z, b ; c ; 1)=1+\sum_{n=1}^{\infty} \frac{(b)_{n}}{(c)_{n}}\left(\frac{z}{n}+\left(\frac{1}{n} \sum_{k=1}^{n-1} \frac{1}{k}\right) z^{2}+\cdots\right) .
$$

Now let

$$
g(z)=\frac{\Gamma(c) \Gamma(c-z-b)}{\Gamma(c-z) \Gamma(c-b)}=1+\sum_{n=1}^{\infty} a_{n} z^{n} .
$$

Differentiating (28) logarithmically, we have

$$
\frac{g^{\prime}}{g}(z)=\sum_{n=0}^{\infty} c_{n} z^{n}
$$

where

$$
c_{n}=\sum_{k=0}^{\infty} \frac{1}{(k+c-b)^{n+1}}-\frac{1}{(k+c)^{n+1}} .
$$

From (28) and (29), we have the recursion formula for $a_{n}$ :

$$
(n+1) a_{n+1}=\sum_{k=0}^{n} a_{n-k} c_{k}, \quad \text { for } n \geq 1 .
$$

In particular, by equating the coefficients of (27) and (28), we obtain

\section{Proposition 2.}

$$
\sum_{n=1}^{\infty} \frac{1}{n} \frac{(b)_{n}}{(c)_{n}}=\sum_{n=0}^{\infty}\left(\frac{1}{n+c-b}-\frac{1}{n+c}\right)
$$

and

$$
\begin{aligned}
\sum_{n=1}^{\infty} \frac{(b)_{n}}{(c)_{n}}\left(\frac{1}{n} \sum_{k=1}^{n-1} \frac{1}{k}\right) \\
\quad=\frac{1}{2}\left\{\sum_{n=0}^{\infty}\left(\frac{1}{n+c-b}-\frac{1}{n+c}\right)^{2}+\sum_{n=0}^{\infty}\left(\frac{1}{(n+c-b)^{2}}-\frac{1}{(n+c)^{2}}\right)\right\} .
\end{aligned}
$$

We add that since $\frac{(b)_{n}}{(c)_{n}}=\frac{\Gamma(c)}{\Gamma(b)}, \frac{\Gamma(n+b-1)}{\Gamma(n+c-1)} \sim \frac{\Gamma(c)}{\Gamma(b)} n^{b-c}$ as $n \rightarrow \infty$. The series (30) and (31) converge absolutely when $b<c$.

We now choose $c=1$ and $b=z$ in (30) and expand both sides as power series at $z=0$. We obtain

$$
\sum_{k=1}^{\infty} z^{k}\left(\sum_{n=k}^{\infty} \frac{s_{k}^{n}}{n \cdot n !}\right)=\sum_{n=1}^{\infty} \frac{(z)_{n}}{n \cdot n !}=\left(\sum_{n=1}^{\infty} \frac{1}{n-z}-\frac{1}{n}\right)=\sum_{k=1}^{\infty} \zeta(k+1) z^{k}
$$

Therefore

$$
\zeta(k+1)=\sum_{n=k}^{\infty} \frac{s_{k}^{n}}{n \cdot n !} .
$$


This identity reveals an interesting relation between the $\zeta(n)$ and the Stirling numbers of the first kind. In particular, for $k=2$, it yields (iv).

Similarly, choosing $c=1$ and $b=z$ in (31) and expanding both sides in terms of power series at $z=0$, we obtain

$$
\begin{gathered}
\left\{\sum_{n=1}^{\infty} \frac{1}{n^{2}}\left(\sum_{k=1}^{n-1} \frac{1}{k}\right)\right\} z+\left\{\sum_{n=1}^{\infty}\left(\frac{1}{n} \sum_{k=1}^{n-1} \frac{1}{k}\right)^{2}\right\} z^{2}+\cdots \\
=\zeta(3) z+\frac{1}{2}\left(\zeta^{2}(2)+3 \zeta(4)\right) z^{2}+\cdots
\end{gathered}
$$

Therefore

$$
\sum_{n=1}^{\infty}\left(\frac{1}{n} \sum_{k=1}^{n-1} \frac{1}{k}\right)^{2}=\frac{1}{2}\left(\zeta^{2}(2)+3 \zeta(4)\right)=\frac{11}{4} \zeta(4),
$$

and this proves (i).

Finally, we state without proof that the application of the same method to Morley's identity [5, p. 301]

$$
1+\sum_{n=1}^{\infty}\left(\frac{(a)_{n}}{n !}\right)^{3}=\cos \left(\frac{\pi a}{2}\right) \frac{\Gamma\left(1-\frac{3 a}{2}\right)}{\left(\Gamma\left(1-\frac{a}{2}\right)\right)^{3}}
$$

yields (iv):

$$
\sum_{n=2}^{\infty} \frac{1}{(n+1)^{3}}\left(\sum_{k=1}^{n} \frac{1}{k}\right)=\frac{1}{4} \zeta(4) .
$$

To end this paper, we note that in [2] the Borweins also proposed to sum the following series involving alternating harmonic terms

$$
\sum_{n=1}^{\infty}\left(\frac{1}{n} \sum_{k=1}^{n}(-1)^{k+1} \frac{1}{k}\right)^{2} \text {. }
$$

Currently, the nature of this sum is not known. We comment that one way to generate the terms involving the alternating series $\sum_{k=1}^{n}(-1)^{k} \frac{1}{k}$ is to consider

$$
\begin{aligned}
\frac{\left(\frac{\alpha}{2}\right)_{n}\left(-\frac{\alpha}{2}+\frac{1}{2}\right)_{n}}{n !\left(\frac{1}{2}\right)_{n}} & =\left(\frac{1}{2 n}\right) \alpha(1-\alpha)\left(1+\frac{\alpha}{2}\right)\left(1-\frac{\alpha}{3}\right) \cdots\left(1-\frac{\alpha}{2 n-1}\right) \\
& =\frac{\alpha}{2 n}+\alpha^{2}\left(\frac{1}{2 n} \sum_{k=1}^{2 n-1}(-1)^{k} / k\right)+\cdots .
\end{aligned}
$$

So to be able to use our approach, we need a version of (1) for the series

$$
{ }_{4} F_{3}\left(\begin{array}{c}
\frac{\alpha}{2},-\frac{\alpha}{2}+\frac{1}{2}, \beta, \gamma ; 1 \\
\frac{1}{2}, b, c
\end{array}\right) .
$$

But this seems highly unlikely. Thus, from the perspective of the hypergeometric series, the problem of determining the exact nature of the sums involving the alternating harmonic terms seems very formidable! 


\section{REFERENCES}

1. B. C. Berndt, Ramanujan's notebooks, part I, Springer-Verlag, New York, 1985.

2. D. Borwein and J. M. Borwein, On some intriguing sums involving $\zeta(4)$, preprint.

3. G. Polya and G. Szegö, Problems and theorems in analysis, Vol. I, Springer-Verlag, New York, 1972.

4. W. Rudin, Real and complex analysis, McGraw-Hill, New York, 1966.

5. E. T. Whittaker and G. N. Watson, $A$ course of modern analysis, 4th ed., Cambridge Univ. Press, 1958.

Department of Mathematics, University of Florida, Gainesville, Florida 32611

E-mail address: shen@math.uf 1. edu 